\title{
Scaling in the space climatology of the auroral indices: is SOC the only possible description?
}

\author{
N. W. Watkins \\ British Antarctic Survey, High Cross, Madingley Rd., Cambridge CB3 0ET, UK \\ Received: 21 December 2001 - Revised: 26 May 2002 - Accepted: 6 June 2002
}

\begin{abstract}
The study of the robust features of the magnetosphere is motivated both by new "whole system" approaches, and by the idea of "space climate" as opposed to "space weather". We enumerate these features for the $A E$ index, and discuss whether self-organised criticality (SOC) is the most natural explanation of the "stylised facts" so far known for $A E$. We identify and discuss some open questions, answers to which will clarify the extent to which $A E$ 's properties provide evidence for SOC. We then suggest an SOC-like reconnection-based scenario drawing on the result of Craig (2001) as an explanation of the very recent demonstration by Uritsky et al. (2001b) of power laws in several properties of spatiotemporal features seen in auroral images.
\end{abstract}

\section{Introduction: the "stylised facts" of the $A E$ indices}

In the last few years, the advent of magnetospheric models (e.g. Chang, 1999; Consolini, 1997; Chapman et al., 1998) based on the idea of self-organised criticality (SOC) (e.g. Jensen, 1998; Sornette, 2000) or more general critical phenomena has stimulated new efforts towards the identification of those features of the magnetosphere which are robust and repeatable in statistical studies taken over arbitrarily long periods of time. This new knowledge, therefore, provides potentially important information for studies of magnetospheric "space climate" (e.g. Boteler, 1991), as distinct from the many case studies of individual magnetospheric events, in particular magnetic storms and substorms, a field increasingly referred to as "space weather". SOC models typically have power law probability density functions (PDFs) and " $1 / \mathrm{f}$ " power spectra (PSDs) for energy release from the system, signals of a multiscale process with no preferred scale in space or time.

In mathematical economics, the robust and repeatable mathematical properties of an economic index or other variable are sometimes referred to as "stylised facts" (e.g.

Correspondence to: N. W. Watkins (nww@bas.ac.uk)
Mikosch and Starica, 2000). In this paper, we seek to establish some of the stylised facts of the magnetosphere, and to sketch out a possible type of simple model for some of its outputs. We have chosen the specific example of the $A E$ indices ( $A E, A U, A L$ and $A O$ ). Our purpose is twofold: first, we wish to collect some still-relevant results on the space climatology of the indices which were obtained before the rise of the SOC paradigm (see also the earlier review of Klimas et al., 1996). New findings in this area tend now to be interpreted exclusively within a framework, such as SOC or other nonequilibrium critical phenomena, such as noise assisted topological phase transitions. It seems to us, however, that such behaviour will be most clearly demonstrable if an alternative framework is developed in parallel that seeks to encompass the data, but does not require avalanching or selfregulation (see, also Freeman et al., 2000b; Watkins et al., $2001 \mathrm{~b}, \mathrm{c}$ ). If and when such a description fails, the manner in which it does so would make much more convincing the need for an SOC (or more generally, a self-regulating) description. Insofar as it works, however, such a description is a useful mathematical tool for quantifying the risk of extremes in the indices, and possibly also in mathematically analogous solar wind quantities.

Second, we wish to reiterate our reasons for thinking that the scale-free behaviour of the auroral indices need not necessarily have the same origin as the scale free behaviour seen in newer observations, most notably those based on ultraviolet images. This is important because the modelling of the indices is of continuing space weather/climate interest, but should neither be restricted by the SOC paradigm nor be a restriction on the construction of magnetospheric SOC models, unless it is actually necessary.

It is worth stressing that such arguments are by no means unique to the present author, or the topic of magnetospheric physics. Freckleton and Sutherland (2001) have recently given a clear statement of the need for the construction of such null models, commenting on a claim of SOC in queues in the UK National Health Service. They illustrate that in this case identical behaviour to that observed is also seen in 

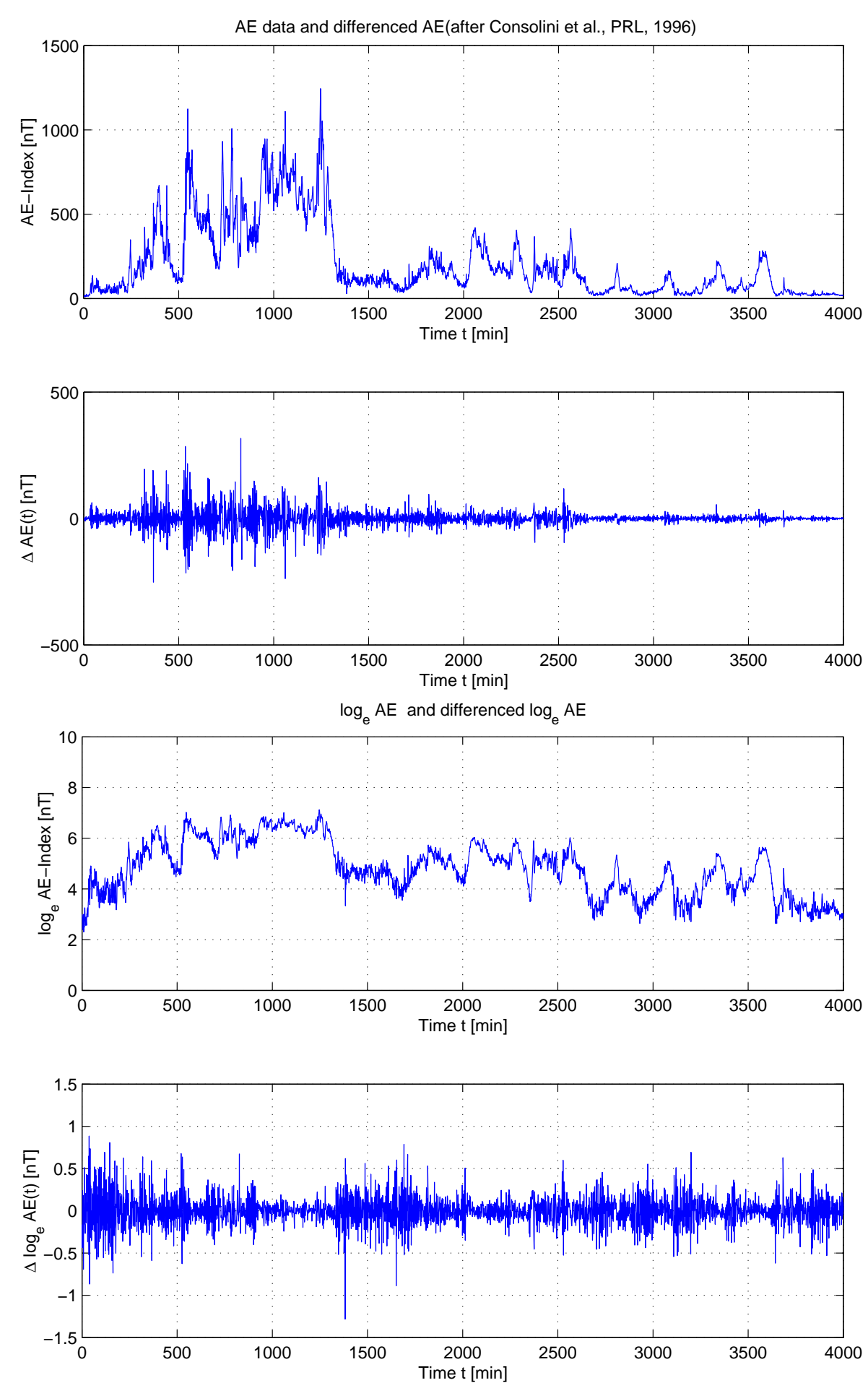

Fig. 1. The top panel (a) shows $A E$ for $4000 \mathrm{~min}$ in 1975, as plotted by Consolini et al. (1996). The second panel (b) shows the first differences of the data, while the third and fourth panels (c) and (d) show the natural logarithm of $A E$ and its first difference. The impulsiveness noted by Consolini et al. (1996) for the data seen in (b) is still present in (d).

(non self-regulating) Brownian motion. More generally, a very clear discussion of the extent to which self-regulating models are needed to explain the ubiquitous scale-free phenomena in natural and man-made systems has been given by Sornette (2000) (see also his recent summary Sornette,
2002). In the plasma context, Krommes (2000) has given an explicit counter-example to the idea that power law tails in time-domain correlation functions are necessarily evidence of SOC. The present paper is an attempt to make some initial developments of this approach in magnetospheric physics, 
extending some of the ideas first expressed by Watkins et al. (2001c) and Chapman and Watkins (2001).

The plan of the paper is as follows: partly because they have been extensively used as proxies for energy dissipation, and thus can be treated as dynamical variables in chaos or SOC-inspired studies, the attributes of the $A E$ indices have been widely studied. In Sect. 1 , we present a critical summary of what has been learned about the indices. We discuss whether SOC is necessary to account for the stylised facts of the indices. In particular, we make the suggestion that $A E$ may be satisfactorily explained as a solar-wind driven component modelled by a multiplicative processes, such as fractional lognormal motion, if coupled with a second component, describing the intrinsic "unloading" behaviour.

In Sect. 2, we discuss several questions that we believe need to be answered more fully in order to clarify the most appropriate model for $A E$. In particular we note that systems with lognormal amplitude distributions, when also exhibiting time domain persistence, will tend to give power law-like PDFs for the burst measures previously applied to $A E$, without the presence of self-organisation, or even truly scale-free amplitude behaviour. We suggest that this kind of "persistent lognormality" is a suitable null model against which SOC should be tested in the auroral indices, and may well have more widespread relevance.

In Sect. 3, we discuss newer auroral imager-derived measurements which promise to identify SOC behaviour more unambiguously, and we draw attention to the existence of a magnetic reconnection-based physical model which may explain some aspects of these most recent results.

Section 4 gives our conclusions. Due to the deliberately narrow focus of this paper, we have not dealt with all of the available literature on SOC in the magnetosphere. Consequently, the reader is encouraged to consult the review papers by Chapman and Watkins (2001) and Consolini and Chang (2001), and also the extended discussions of the evidence for SOC and/or SOC-like multiscale behaviour in the magnetosphere given by Chang (1999), Klimas et al. (2000), Lui (2002) and Sitnov et al. (2002).

\subsection{Time series and PDF}

$A E$ estimates maximal ionospheric current densities via the upper $(A U)$ and lower $(A L)$ envelopes of magnetic perturbations and is usually derived from 12 magnetometers underneath the mean position of the auroral electrojets. The total envelope is $A E=A U-A L$. In Fig. 1a, we show an example of three days of the $A E$ time series in early 1975, as studied by Consolini et al. (1996). As noted by Consolini et al. (1996), the first difference of $A E$ (Fig. 1b) shows apparent "burstiness". However, a fractional lognormal motion (flm) - a fractional Brownian motion (e.g. Malamud and Turcotte, 1999), with a lognormal rather than a Gaussian distribution of amplitudes - would also show such burstiness. More significantly, the first difference of $\log _{e} A E$ is also bursty, whereas an flm would show a normal distribu-

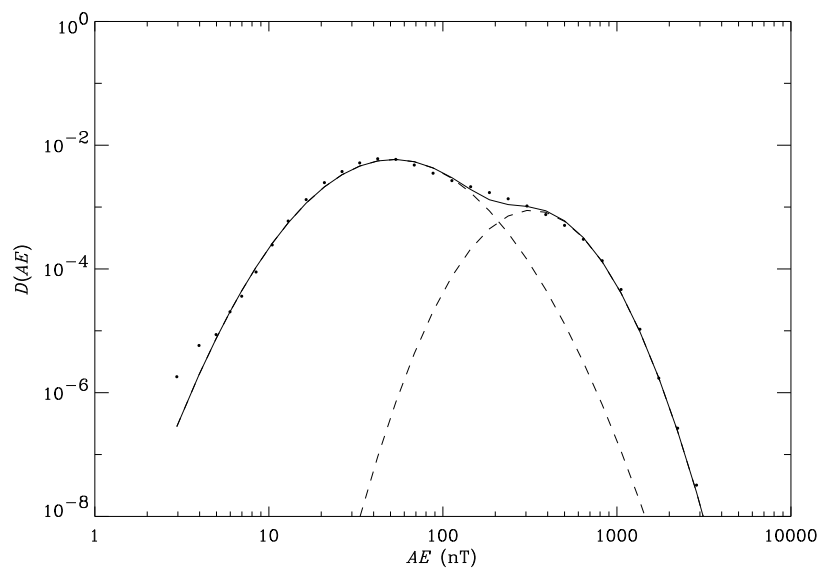

Fig. 2. A bi-lognormal fit to the PDF of $A E$ for the period January 1978 to June 1978.

tion in that quantity (see Fig. 6). This suggests that a single fractional lognormal motion would fail as a model of $A E$.

This conclusion is consistent with the fact that Consolini and de Michelis (1998) found $A E$ to have a two component probability distribution function (PDF), well described by two quasi-lognormal distributions, one of which had an exponential cutoff. A similar result was shown by Vassiliadis et al. (1996) for $|A L|$. In Fig. 2, we show that two lognormals, with the standard prefactor and without exponential modification, give a very good fit to the PDF of $A E$ for the period January 1978 to June 1978.

\subsection{Second order statistics: Fourier spectrum and ACF}

While the range of amplitudes present in $A E$ is described by the PDF, one may study $A E$ in the frequency domain by Fourier analysis. Tsurutani et al. (1990) used the Welch averaged periodogram to obtain a power spectral density for 5-min $A E$ data for the periods 1978-1980 and 1967-1970. The "broken power law" spectrum they obtained is typical of $A E$ even for shorter runs of data (see, e.g. Fig. 3, where Tsurutani et al.'s approach is applied to 1 year of $A E$ data). The $1 / f$ behaviour is seen essentially all the time, but Consolini (1999) has shown that the higher frequency $1 / f^{2}$ component is present for high activity levels.

The power spectral density can be described in terms of two components, one of which (the " $1 / f$ " part) indicates long-range correlation in time.

The autocorrelation function (ACF) of a time series $X(t)$

$A C F(\tau)=\sum_{t} X(t) X(t+\tau)$

allows one to express the above behaviour in the time rather than the frequency domain. Takalo et al. (1993) found an ACF with qualitatively two-component behaviour (see Fig. 4 which is a replotting of their Fig. 1 with the data they used). The long-range tail becomes progressively clearer as one observes longer and longer (20 days or more) time series, 


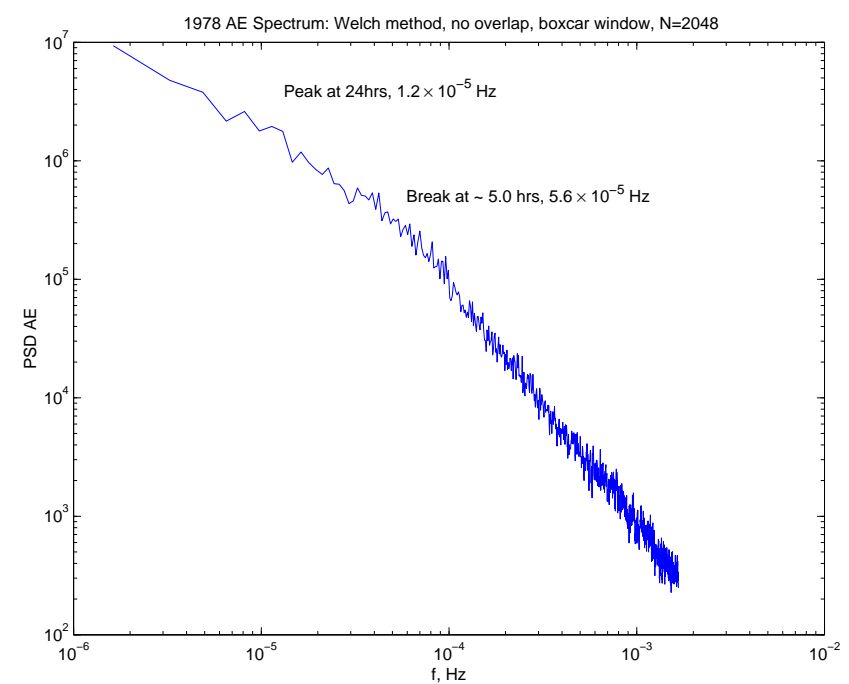

Fig. 3. PSD for $A E$ using same approach as that in Tsurutani et al. (1990) but for 1 year rather than 3. A similar spectrum is seen over the shorter period, though the 24-h peak is not clear in only one year's worth of data.

though in Fig. 4, we follow Takalo et al. (1993) by plotting only lags up to $500 \mathrm{~min}$. Even for short runs of data (5 days), however, a fast-dropping exponential ACF is seen, for which the normalised amplitude halves in about $100 \mathrm{~min}$. As noted by Mantegna and Stanley (2000), "fast decaying autocorrelation functions and power spectra resembling white noise (or " $1 / f$ " " power spectra for the integrated variable) are "fingerprints" of short-range correlated stochastic processes." Two classic examples of such processes are the velocity of a Brownian particle (e.g. Mantegna and Stanley, 2000) and the "random telegraph" (a Poisson-switched onoff pulse train, e.g. Jensen, 1998).

In order to further examine the time-domain behaviour of the $A E$ series, Takalo et al. (1993) studied the second order structure function $S_{2}(\lambda \Delta t)$. This is defined as

$S_{2}(\lambda \Delta t)=\langle X(t+\lambda \Delta t)-X(t)\rangle^{2}$

with $\langle\ldots\rangle$ denoting a time average. On the assumption of stationarity, this equates to $2\left(\langle X(t)\rangle^{2}-A C F(\lambda \Delta t)\right)$. A selfaffine (e.g. Malamud and Turcotte, 1999; Sornette, 2000) signal has $S_{2}(\lambda \Delta t) / S_{2}(1) \sim \lambda^{2 H}$ (Takalo et al., 1993) and so a $\log$-log plot of $\left(S_{2}(\lambda \Delta t) / S_{2}(1)\right)^{1 / 2}$ versus $\lambda$ for such a selfaffine signal would give a slope equal to $H$. However, as we have discussed elsewhere (Watkins et al., 2001b), an exponential form for the ACF, whatever its cause, must necessarily imply a region of $H=0.5$ scaling in the structure function. The Taylor series expansion of the exponential means that the inferred $H$ value must be 0.5 (Watkins et al., 2001b), and so the nearly linear scaling region with $H \sim 0.5$ seen by Takalo et al. (1993) might be just another manifestation of the observed exponential autocorrelation, rather than in itself necessarily implying that the high frequency part of the $A E$ signal is a scale free coloured noise.

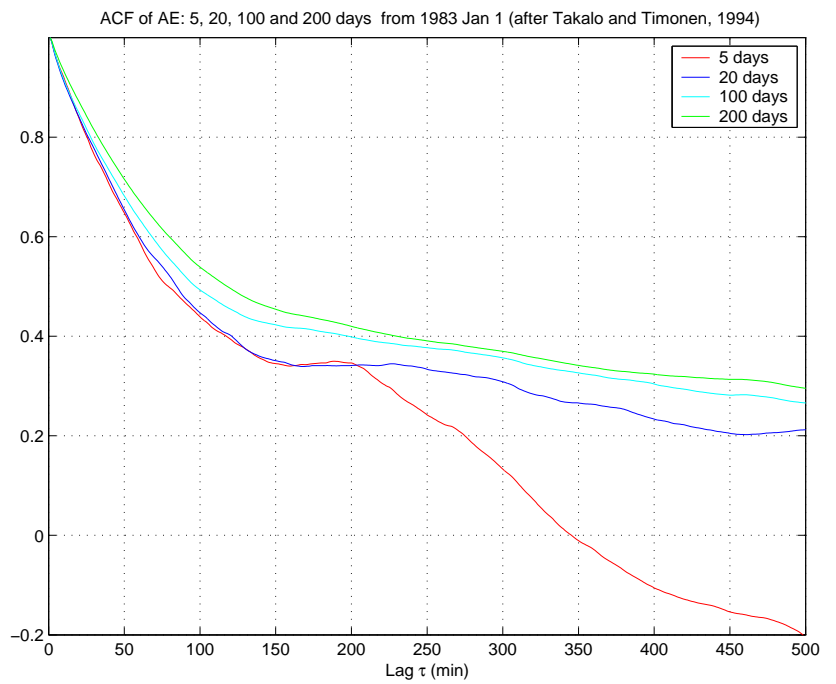

Fig. 4. After Takalo et al. (1993), comparison of autocorrelation functions of $A E$ taken over 5, 20, 100 and 200 days in 1983.

\subsection{Burst distributions: duration, size and waiting time}

The advent of the idea of self-organised criticality led Takalo (1993) and Consolini (1997) to study "bursts" in the $A E$ index time series. These bursts were defined using a constant threshold method whereby the set $\left\{t_{1}\right\}$ denotes all the upcrossings of a given threshold, and the set $\left\{t_{2}\right\}$, all the downcrossings, so burst "size" $s$ is given by

$s=\int_{t_{1}}^{t_{2}} A E(t) d t$

while "durations" and "waiting times" are given by the intervals between a given $t_{1}$ and the next $t_{2}$, and that between a $t_{2}$ and the next $t_{1}$, respectively. The first studies of the probability density function (PDF) of $s$ were both on a single year's worth of data, and the resulting distributions of $s, \tau$ and $T$ were all power laws with an exponential roll off. Study of longer time series later showed, however (e.g. Consolini, 1999; Freeman et al., 2000a), that the scale-free behaviour was interrupted by a bump in both the burst size and duration PDFs. The simplest interpretation of this is that there are two components in $A E$, presumably the same two components seen in the PSD and PDF of $A E$ itself, although this raises several questions which will be discussed below (see also the extended discussions in Freeman et al., 2000a; Watkins et al., 2001b).

If there are effectively two components to the $A E$ time series, then a natural question is, "Where do they come from?" Due to the long-established evidence for the driving of the $D P 2$ convection currents (e.g. Kamide and Baumjohann, 1993) (believed to be the most continuous contributor to the $A E$ index) by the solar wind, Freeman et al. (2000a) performed an identical constant threshold burst duration analysis on the solar wind $v B_{s}$ and $\epsilon$ functions, and also on the $A U$ and $A L$ indices. It was found that burst lifetimes in all 


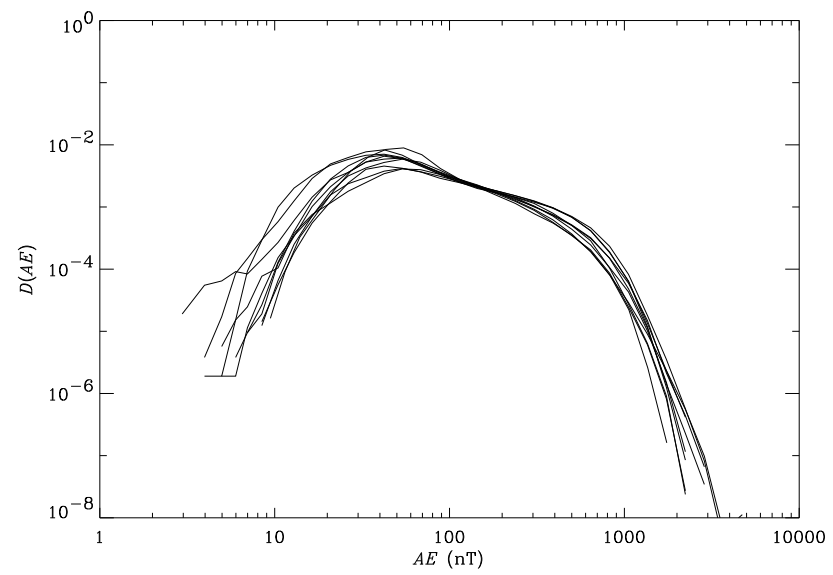

Fig. 5. Each curve is the PDF of $A E$ taken over one of the years from 1978 to 1988.

four quantities showed power laws with very similar slopes. However, the aforementioned bump in the burst duration signal was seen only in the magnetospheric signals. Since the bump was larger in $|A L|$ (mainly drawn from post-midnight stations) than $A U$ (mainly dusk), Freeman et al. (2000a) inferred that the bump was the signature of the substorm $D P 1$ current. The inference drawn from this study was that the scale-free component in $A E$ was most probably a consequence of scale-free behaviour in the solar wind, and that the "bump" was an objective identification of the substorm with a characteristic time scale.

A number of important criticisms may be raised of the result, however. Obviously it was not ideal to use a noncontemporary time series (1978-1988 for the $A U$ and $A L$ indices and 1995-1998 for the solar wind data). Unfortunately, no two simultaneous data sets of many years' in length were available for $A U / A L$ and the solar wind data. This problem was addressed in part by a comparison between the burst duration PDFs taken from a 4-year period of the $A U$ and $A L$ series and the existing solar wind burst PDFs from an equivalent phase of the solar cycle. No significant difference was seen between the burst PDFs taken from this section and those from a full 10-year series of $A E$. The main justification for the approach used, however, is the remarkable stability of the bi-lognormal aspect of the underlying PDF of $A E$. While the curves vary in parameters, they can be fitted consistently by bi-lognormal fits. In consequence, if, as we suspect, the power laws found are partially a consequence of the " $1 / \mathrm{x}$ " power law scaling region present in a lognormal distribution (Sornette, 2000), regardless of its parameters, they will be stable from year to year. Each curve in Fig. 5 is the PDF of $A E$ taken over one of the years from 1978 to 1988. A similar stability was found by Burlaga and Lazarus (2000) in PDFs of the solar wind velocity, density and temperature during the period from which the WIND measurements were taken.

In addition, to eliminate the effect of the angle $\theta$, which represents the solar wind-magnetosphere coupling process in the $\epsilon$ function, Freeman et al. (2000b) subsequently exam- ined the PDFs for burst durations, waiting times and sizes in the radial solar wind Poynting flux. Both durations and waiting times could be fitted by identical exponentially-truncated power laws, while the apparent power law region in burst size is also reminiscent of long-ranged lognormal distributions (Malamud and Turcotte, 1999; Sornette, 2000).

More recently, Uritsky et al. (2001a) have examined a contemporary pair of data sets for solar wind $\epsilon$ and $A E$ (although the series are shorter (6 months) and with a 5-min resolution). They found inter alia different scaling behaviours for timeaveraged activity and event survival probability in the two signals, and an apparent break in the scaling of the dependence of $A E$ burst size on duration. They interpreted their results as evidence of a distinct internal magnetospheric dynamical component in the $A E$ signal.

\subsection{Fractional lognormal motion and "persistent lognor- mality"}

It is straightforward to illustrate how some of the above conjectures can be checked by using a numerical realisation of fractional lognormal noise. The details will be presented elsewhere, but here we sketch how such a process may be used to approximate the " $1 / f$ " component of the $A E$ series. We postpone to a subsequent work the implementation of a suitable model of the second unloading component that has been postulated above, but note that a first order approximation to the " $1 / f^{2}$ " term was presented by Watkins et al. (2001b). We made a time series of fractional lognormal motion with zero mean and unit standard deviation, and spectral exponent -1.5 , using the algorithm of Malamud and Turcotte (1999). Taking the spacing of the data to be $1 \mathrm{~min}$, we plot 4000 points from the resulting time series in Fig. 6 . From a longer series we have confirmed that power law PDFs for size, duration and waiting times of bursts, as defined in, for example, Consolini (1997), do indeed result from such a model, while we also see size-duration relations, with scale breaks, qualitatively similar to those seen by Uritsky et al. (2001a).

The power spectrum and amplitude distribution of $Y$ are, by construction, $f^{-1.5}$ and lognormal, while perhaps more surprisingly, the differenced time series $X$ has leptokurtic tails and shows a range over which a scaling collapse of the type described by Hnat et al. (2002) can be demonstrated. However, agreement with observed autocorrelation functions and Hurst exponent measures is relatively poor, suggesting that we need to include a representation of the unloading component before a useful comparison can be made.

We note that fractional lognormal motion has several competitors as a model of real anomalous diffusion, most notably truncated Levy flights, and continuous time Levy flights, the latter being generalised random walks in which Levy stable increments are combined with a random distribution of times between steps (Paul and Baschnagel, 1999). We plan to investigate these alternatives to find an optimum description of the driven component of $A E$. 

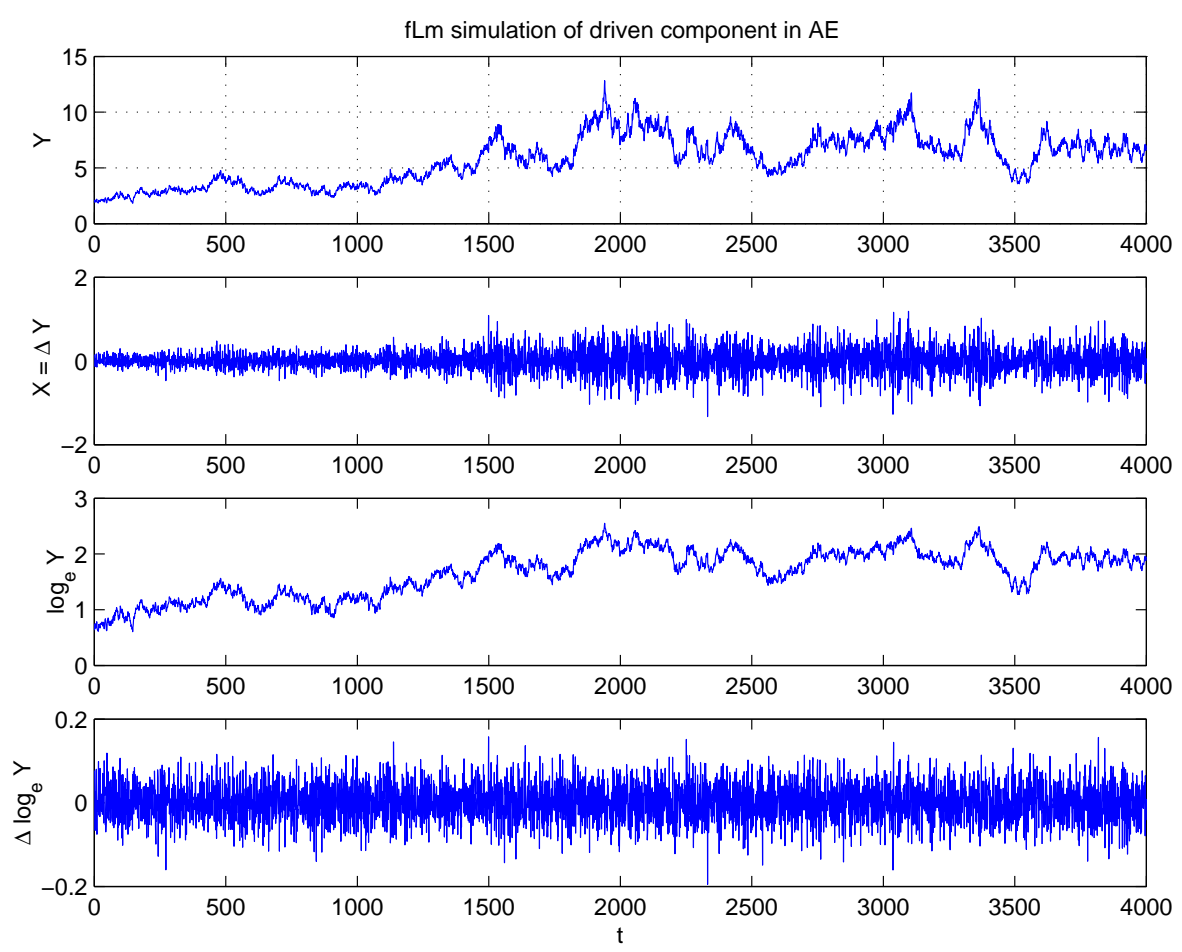

Fig. 6. The top panel shows a realisation of 4000 points of a synthetic $A E$ "driven component" $Y$, with lognormal amplitude distribution and a power spectral density $\sim f^{-1.5}$. The second panel shows the first difference (compare Fig. 1b), the third panel shows the natural log of $Y$ (compare Fig. 1c), while the fourth panel shows the differenced $\log Y$ time series, in which the intermittency apparent in the second panel has disappeared.

\subsection{Summary of the "stylised facts" of $A E$}

We may summarise the above non-exhaustive collection of "stylised facts" about $A E$, with some tentative interpretations, as follows:

1. The $A E$ time series has more than one component (see also, for example, Table 5.2 in Kamide and Baumjohann, 1993).

2. If $A E$ has more than one component, it may not necessarily mean that more than one process is at work. For example, Chapman et al. (1998) showed that a sandpile model could produce both a scale-free and non-scalefree component, where the non-scale-free component is identified with the systemwide events in the model).

3. At least one of the components in $A E$ is long-range correlated (and fractal) in time.

4. This observed long-range correlation may originate in that present in the solar wind Poynting flux, a hypothesis supported by the identical power laws is seen both in waiting time and burst duration by Freeman et al. (2000b), as expected for the isosets of a fractal random walk (e.g. Watkins et al., 2001a).

5. One component apparently has a characteristic time scale, which Freeman et al. (2000a) identify with the substorm.
6. The PDF of the $A E$ signal itself is apparently not scale free, but rather is well described by a bi-lognormal. However, it is already known that such distributions are also very good approximations to underlying multifractal cascades (see, for example, the discussion in Burlaga, 2001), and so, since evidence for multifractality in $A E$ exists (e.g. Consolini et al., 1996), we admit that a fractional lognormal noise component may be a less well motivated choice of model for the long-range correlated part of $A E$ than it might at first seem.

7. Nonetheless, it is possible that the power law PDF for burst durations and waiting times results simply because one part of the $A E$ signal is well approximated by fractional lognormal noise. Consequently, the less clear cut scaling in burst size would then result from the convolution (implicit in the method used to define bursts) between the non-scale-free amplitude PDF and the genuinely scale-free persistent property of the $A E$ time series.

\section{Outstanding questions}

The long-range time correlation and long tailed PDFs seen in $A E$ can be physically motivated by sporadic, localised energy release events in the magnetotail, and can be modelled by sandpile algorithms (e.g. Chapman et al., 1998). The ob- 
served two-component property discussed above can also be accounted for, at least if we make the hypothesis of a difference between systemwide and internal energy release events. However, a number of issues arise from the summary presented above, and it is still the view of the present author that more definitive answers to the following questions are needed, to establish more clearly if SOC is a necessary explanation for the behaviour of $A E$ rather than a possible one.

\subsection{Does time correlation imply SOC?}

The answer to this would seem to be "no", in that one may have long-range correlation in time, signalled by " $1 / f$ " spectra, in many other classes of process, some of which are not spatially long-range correlated at all. Conversely, some (but not all) SOC models (most notably the original BTW model; Bak et al., 1987) do not exhibit long-range time correlation in their outputs. Consequently, it is neither advisable to test for SOC relying only on " $1 / f$ " spectra, nor to use the power law waiting times seen in shell models as a test against SOC (see also Watkins et al., 2001a, and references therein where this issue is discussed in reference to solar flares, and the discussion in the context of fusion plasmas of Krommes, 2000).

2.2 Is the observed amplitude (rather than burst) PDF in $A E$ consistent with criticality?

The problem with interpreting power laws in burst size as evidence of scale-free behaviour is that one may also have a time-correlated lognormal process (see, for example, the simulations presented in Fig. 19 of Malamud and Turcotte (1999), and the illustrative time series shown in Fig. 6). Integrating a times series of the amplitude of such a process in the region where it mimics a power law (e.g. Figs. 4.2 and 4.3 in Sornette, 2000) gives rise to a power law-like burst distribution, provided that the signal is persistent in time. In fact, even burst distributions constructed from a Gaussian persistent process are remarkably power law-like (see Fig. 2 of Watkins et al., 2001a). Thus, we currently need to consider "persistent lognormality", as well as SOC as an alternative explanation for the stylised facts of $A E$ bursts.

2.3 Can power spectra be reconciled with burst distributions?

It has been remarked (Channon Price, personal communication, 1999) that the monofractal nature of the burst duration distributions seen would seem hard to reconcile with the apparent biaffine nature of the power spectrum of $A E$. This is because a mono-fractal (or bifractal) should give the same $H$ value (or values) to both first order and second order measures. The resolution of this apparent paradox is that a mixed signal, which has a fractal part and a non-fractal part, does not have this limitation. A first order measure, such as a burst duration, basically measures the length of a fractal curve (because the slope of the isoset distribution is governed by the fractal dimension of the curve which is crossing the threshold). If a signal has a fractal component which accounts for most of the curve, it may take a long time series for the nonfractal part to become apparent. In the author's view, this is the reason why the bump in $A E$ burst sizes needed a long time series (as used by Consolini, 1999) to become apparent, rather than the 1-year series studied by Consolini (1997).

A second order measure, such as $S_{2}$, conversely, measures the distribution of variance in a signal, due to the formal equivalence between the information in $S_{2}$ and that in the ACF or power spectrum. A sharp change in level in the time series caused by a non-fractal substorm component can be revealed by the $1 / f^{2}$ power spectrum it gives rise to at high frequencies. The presence of such a non-fractal component explains, in contrast with first order methods, why the power spectrum reveals a spectral break in even as few as 4 days of $A E$ data, and it is clearly apparent in Fig. 3 taken from 1 year of $A E$ data.

\subsection{How much of the time correlation in $A E$ comes from the solar wind?}

It seems to be increasingly accepted (e.g. Takalo et al., 2000; Price and Newman, 2001; Uritsky et al., 2001a) that some part of the scale-free behaviour of the $A E$ signal comes from the scale-free solar wind driver. However, two caveats need to be noted. One is that, as with $A E$, the solar wind probably also has a multifractal character, and so comparison of fractal dimensions, as in Freeman et al. (2000a) between the solar wind and $A U / A L$, is really just comparing measures at one order. However, Vörös et al. (1998) showed that the higher order structure functions of low pass filtered, solar wind magnetic field fluctuations and those for an unfiltered ground-based magnetometer signal were also in close agreement. More importantly, the Hurst exponent for a signal is its roughness averaged over many length scales. Even if the $A E$ output is nonlinearly driven by the solar wind, similar roughness values in the output and the driver would not require individual bursts to be the same. Consequently, the absence of one-to-one mappings between integrated input power and $A E$ bursts, as found by Uritsky et al. (2001a), may not be so surprising. We will return to this point in future work.

\section{Further motivation for criticality or SOC behaviour}

For all the above reasons, better indicators of SOC behaviour than those derived from $A E$ are needed in the magnetospheric case. Uritsky et al. (2001b) have recently shown remarkably clear power laws in several burst measures drawn from time-evolving "blobs" seen by the UVI auroral imager on the WIND satellite. While more studies are necessary, a particularly interesting result is the $E^{-1.5}$ power law that they observe in the time integrated energy of blobs. Following Uritsky et al. (2001b) in assuming that the blobs they observed in UVI data correspond to magnetic reconnection events in the magnetotail, it is tempting to explain the observed energy spectrum using the results of Craig (2001). He was considering simple models of solar flares and showed 
that an $E^{-1.5}$ power law is exactly the spectrum to be expected from distributed reconnection events with $2 \mathrm{D}$ current sheet geometry, using the known properties of analytic solutions for reconnection and a relatively conservative set of additional assumptions. The optical observations of Uritsky et al. (2001b) thus suggest SOC, or least more obviously, SOClike behaviour more directly than $A E$. They illustrate clearly how studying different physical quantities or measures will help to better answer the question of whether SOC is present in the magnetosphere.

\section{Conclusions}

SOC is not yet needed to explain the properties of $A E$ 's time series: at least where the PDF, power spectrum, structure function and "burst statistics" are concerned. Nonetheless, because SOC is an economical and physically motivated approach to capturing these properties, further studies are needed to clarify some outstanding issues, in particular the relationship between solar wind scaling and that seen in $A E$. A recent pointer to how the SOC investigations may be advanced is the demonstration of power law scaling in spatiotemporal events in a signal (UVI images), which may be less ambiguous than the necessarily "mixed" signal seen by $A E$.

Acknowledgements. This paper is based on an invited talk given at the IAGA meeting in Hanoi, Vietnam, August 2000. N. W. Watkins thanks the conveners of this session for the opportunity to give this talk, and their dedicated efforts at the meeting. He would also like to thank M. Freeman, and the participants in the 2002 Venice Workshop on Complexity in the Earth's Magnetospheric Dynamics for innumerable stimulating discussions; D. Riley for his work in preparing Figs. 2 and 5; and V. Uritsky and A. Klimas for a preprint of Uritsky et al. (2001b).

\section{References}

Bak, P., Tang, C., and Wiesenfeld, K.: Self-organized criticality an explanation of $1 / f$ noise, Phys. Rev. Lett., 59, 381-384, 1987.

Boteler, D.: Predicting geomagnetic disturbances on power systems, EOS, Trans. Amer. Geophys. Union, 72, 159-160, 1991.

Burlaga, L. F. and Lazarus, A. J.: Lognormal distributions and spectra of solar wind plasma fluctuations: Wind 1995-1998, J. Geophys. Res., 105, 2357-2364, 2000.

Burlaga, L. F.: Lognormal and multifractal distributions of the heliospheric magnetic field., J. Geophys. Res., 106, 15917-15927, 2001.

Chang, T. S.: Self-organized criticality, multi-fractal spectra, sporadic localized reconnections and intermittent turbulence in the magnetotail, Phys. Plasmas, 6, 4137-4145, 1999.

Chapman, S. C. and Watkins, N. W.: Avalanching and selforganised criticality, a paradigm for geomagnetic activity?, Space Sci. Rev., 95, 293-307, 2001.

Chapman, S. C., Watkins, N. W., Dendy, R. O., Helander, P., and Rowlands, G.: A simple avalanche model as an analogue for magnetospheric activity, Geophys. Res. Lett., 25, 2397-2400, 1998.
Consolini, G.: Sandpile cellular automata and magnetsopheric dynamics, in: Cosmic physics in the year 2000, (Eds) Aeillo, S., et al., Proceeding vol. 58, Societa Italia de Fisica, 1997.

Consolini, G.: Punctuated equilibrium, 1/f noise, and metastability in magnetospheric dynamics, EOS, Trans. Amer. Geophys. Union, S272, 27 April 1999.

Consolini, G. and de Michelis, P.: Non-Gaussian distribution function of $A E$-index fluctuations: evidence for time intermittency, Geophys. Res. Lett., 25, 4087-4090, 1998.

Consolini, G., Marcucci, M. F., and Candidi, M.: Multifractal structure of auroral electrojet data, Phys. Rev. Lett., 76, 4082-4085, 1996.

Consolini, G. and Chang, T. S.: Magnetic field topology and criticality in geotail dynamics: relevance to substorm phenomena, Space Sci. Rev., 95, 309-321, 2001.

Craig, I. J. D.: A reconnection model for the distribution of flare energies, Sol. Phys., 202, 109-115, 2001.

Freeman, M. P., Watkins, N. W., and Riley, D. J.: Evidence for a solar wind origin of the power law burst lifetime distribution of the $A E$ indices, Geophys. Res. Lett., 27, 1087-1090, 2000a.

Freeman, M. P., Watkins, N. W., and Riley, D. J.: Power law distributions of burst duration and interburst interval in the solar wind: turbulence or dissipative self organised criticality?, Phys. Rev. E, 62, 8794-8797, 2000b.

Freckleton, R. P. and Sutherland, W. J.: Hospital waiting lists - do power laws imply self-regulation?, Nature, 413, 382, 2001.

Hnat, B., Chapman, S. C., Rowlands, G., Watkins, N., and Farrell, W.: Finite size scaling in the solar wind magnetic field energy density as seen by WIND, Geophys. Res. Lett., 29, 86-1-86-4, 2002.

Jensen, H. J.: Self-organised criticality: emergent complex behaviour in physical and biological systems, Cambridge University Press, Cambridge, 1998.

Kamide, Y. and Baumjohann, W.: Magnetosphere-Ionosphere Coupling, Springer, Berlin, 1993.

Klimas, A. J., Vassiliadis, D., Baker, D. N., and Roberts, D. A.: The organised nonlinear dynamics of the magnetosphere, J. Geophys. Res., 101, 13 089-13 113, 1996.

Klimas, A. J., Valdivia, J. A., Vassiliadis, D., Baker, D. N., Hesse, M., and Takalo, J.: Self-organized criticality in the substorm phenomenon and its relation to localized reconnection in the magnetospheric plasma sheet, J. Geophys. Res., 105, 18 765-18 780, 2000.

Krommes, J. A.: Self-organized criticality, long-time correlations, and the standard transport paradigm, Phys. Plasmas., 7, 1752$1758,2000$.

Lui, A. T. Y., Multiscale phenomena in the near-Earth magnetosphere, J. Atmos. Sol.-Terr. Phys., 64, 125-143, 2002.

Malamud, B. D. and Turcotte, D. M.: Self-affine time series: I. generation and analyses, Adv. Geophys., 40, 1-87, 1999.

Mantegna, R. N. and Stanley, H. E.: An introduction to econophysics: correlations and complexity in finance, Cambridge University Press, 2000.

Mikosch, T. and Starica, C.: Is it really long memory we see in financial returns?, in: Extremes and Integrated Risk Management, (Ed) Embrechts, P., Risk Books, London, 2000.

Paul, W. and Baschnagel, J.: Stochastic processes: from physics to finance, Springer, Berlin, 1999.

Price, C. P. and Newman, D. E: Using the R/S statistic to analyse AE data, J. Atmos. Sol.-Terr. Phys., 63, 1387-1397, 2001.

Sitnov, M. L., Sharma, A. S., Papadopoulos, K., and Vassiliadis, D.: Modelling substorm dynamics of the magnetosphere: From 
self-organization and self-organized criticality to nonequilibrium phase transitions, Phys. Rev. E 65, 016116, 2002.

Sornette, D.: Critical phenomena in the natural sciences - chaos, fractals, selforganization and disorder: concepts and tools, Springer, Berlin, 2000.

Sornette, D.: Mechanisms for powerlaws without self-organization, Int. J. Mod. Phys. C, 13, 133-136, 2002.

Takalo, J.: Correlation dimension of $A E$ data, Ph. Lic. Thesis, Laboratory Report 3, Department of Physics, University of Jyvaskyla, 1993.

Takalo, J., Timonen, J., and Koskinen, H.: Correlation dimension and affinity of $A E$ data and bicolored noise, Geophys. Res. Lett., 20, 1527-1530, 1993.

Takalo, J., Mursula, K. and Timonen, J.: Role of the driver in the dynamics of a coupled-map model of the magnetotail: Does the magnetosphere act as a low-pass filter?, J. Geophys. Res., 105, 27 665-27 672, 2000

Tsurutani, B., Sugiura, M., Iyemori, T., et al.: The nonlinear response of $A E$ to the IMF $B_{S}$ driver: a spectral break at $5 \mathrm{~h}$, Geophys. Res. Lett., 17, 279-282, 1990.

Uritsky, V. M., Klimas, A., and Vassiliadis, D.: Comparative study of dynamical critical scaling in the auroral electrojet index ver- sus solar wind fluctuations, Geophys. Res. Lett., 28, 3809-3812, 2001a.

Uritsky, V. M., Klimas, A., Vassiliadis, D., Chua, D., and Parks, G.: Scale-free statistics of spatiotemporal auroral emissions as depicted by POLAR UVI images: the dynamic magnetosphere is an avalanching system, J. Geophys. Res., in press, 2001b.

Vassiliadis, D., Klimas, A. J., Baker, D. N., and Roberts, D. A.: The nonlinearity of models of the $v B$ (south)- $A L$ coupling, J. Geophys. Res., 101, 19779-19787, 1996.

Vörös, Z., Kovács, P., Juhász, Á., et al.: Scaling laws from geomagnetic time series, Geophys. Res. Lett., 25, 2621-2624, 1998.

Watkins, N. W., Oughton, S., and Freeman, M. P.: What can we infer about the underlying physics from burst distributions observed in an RMHD simulation?, Planet. Space Sci., 49, 12331237, 2001a.

Watkins, N. W., Freeman, M. P., Rhodes, C. S., and Rowlands G.: Ambiguities in determination of self-affinity in the $A E$-index time series, Fractals, 9, 471-480, 2001b.

Watkins, N. W., Freeman, M. P., Chapman, S. C., and Rowlands G.: Testing the SOC hypothesis for the magnetosphere, J. Atmos. Sol.-Terr. Phys., 63, 1435-1445, 2001c. 